\title{
Impact of pulmonary rehabilitation in patients with interstitial lung disease in Singapore
}

\section{Dear Editor,}

Interstitial lung diseases (ILD) encompass a heterogeneous group of lung parenchymal disorders. ${ }^{1}$ ILD-related symptoms impact significantly on quality of life (QoL). ${ }^{2}$ Dyspnoea is the most important factor determining health-related QoL in ILD; contributing factors include reduced exercise capacity, loss of mental well-being and social isolation. ${ }^{3}$

Pulmonary rehabilitation (PR) in ILD can improve exercise capacity without major adverse outcomes., The King's Brief Interstitial Lung Disease (KBILD) questionnaire was developed by Birring et al. as an ILD-specific tool for patient assessment, and measures performance across psychological; breathlessness and activity; and chest symptoms domains. ${ }^{6}$ The KBILD questionnaire has been validated in various European settings. ${ }^{7}$ In Singapore, data on PR are scarce and no disease-specific instrument for ILD has been studied. We describe a single-centre, prospective, observational study examining characteristics and outcomes of ILD patients who have and have not undergone PR. We also describe the use of the KBILD questionnaire in measuring patient-reported outcomes. We obtained the KBILD and permission for its use from Professor Birring in October 2018.

An ILD clinic was established in Singapore General Hospital in 2012. Patients evaluated at this clinic who were aged above 21 years old, diagnosed with any ILD of any severity, and willing to give informed consent were enrolled into a prospective database for our study that was approved by institutional review board.

The KBILD was first administered in English to all ILD patients seen on either their first or second visit after 17 October 2018. Patients who could not selfadminister the KBILD in English were assisted by a translator. The same translator would assist during repeat visits. Follow-up intervals and management decisions were decided by the managing physician. All patients were offered PR. Patients who agreed to PR were assessed by a physiotherapist and a 6-minute walk test (6MWT) was conducted if there were no contraindications. Thereafter, patients were assigned to twice-weekly outpatient PR sessions for 6-8 weeks or home-based exercises. A repeat KBILD questionnaire was administered at subsequent clinic visits. Patients who completed outpatient PR underwent a repeat
6MWT. The primary outcome measure was the KBILD score. Secondary outcome measures included 6MWT distances for patients who completed outpatient PR. Statistical analysis was performed using Stata version 15.0 (StataCorp LLC, College Station, US).

From 17 October 2018 to 31 December 2019, repeat KBILD scores were obtained for 74 patients; 7 who did not provide consent were excluded. Participants' mean age was 65.8 years and $58.1 \%$ were men. There were $86.5 \%$ patients having dyspnoea at presentation and the median modified medical research council (MMRC) score was 1. The characteristics and KBILD scores of these subjects are described in Table 1.

There were 19 patients who completed at least 1 session of PR. Of these, 8 completed 6-8 weeks of twice-weekly outpatient PR and 55 patients declined PR. In the 19 patients who attended PR, KBILD psychological, dyspnoea and chest symptoms scores improved by a mean of $7.3,8.1$ and 1.5 points, respectively. The differences in the change in KBILD scores between visits for patients who participated in PR and patients who declined were statistically significant.

As the PR and non-PR groups were unbalanced, 19 subjects who underwent PR were randomly matched by sex for 19 controls in a case-control model. There continued to be no significant differences between cases and controls in terms of demographics, comorbidities, pulmonary function, diagnoses or treatments received. The 8 patients who completed the $6 \mathrm{MWT}$ before and after PR showed a mean improvement in the 6MWT distance of $45.6 \mathrm{~m}$.

To the best of our knowledge, we describe the first Singapore study in ILD patients where PR improves exercise tolerance, symptoms, and QoL measured, using a patient-reported instrument. The minimal clinically important difference (MCID) in the KBILD is a change of 5 points in the total score, and 6, 7 and 11 points for psychological, breathlessness, and chest symptoms domains, respectively. ${ }^{8}$ Our patients who participated in PR showed an improvement in total, dyspnoea and psychological symptoms scores that were greater than the MCID. Patients who underwent a 6MWT before and after PR demonstrated an improvement in 6MWT distances that was greater than the MCID, which has been established to be 24-45m. ${ }^{9}$ The use of systemic corticosteroids, steroid- 
Table 1. Clinical characteristics, lung function and KBILD scores of patients followed up at an interstitial lung disease clinic in Singapore, grouped by participation in pulmonary rehabilitation

\begin{tabular}{|c|c|c|c|c|}
\hline & $\underset{\mathrm{n}=74}{\text { All }}$ & $\begin{array}{l}\text { Declined to participate in PR } \\
\qquad \mathrm{n}=55\end{array}$ & $\begin{array}{c}\text { Participated in PR } \\
n=19\end{array}$ & $P$ value \\
\hline \multicolumn{5}{|l|}{ Demographics } \\
\hline Age, mean (SD), years & $65.8(10.6)$ & $64.4(10.7)$ & $69.9(9.3)$ & 0.05 \\
\hline Male sex, no. (\%) & $43(58.1)$ & $29(52.7)$ & 14 (73.7) & 0.110 \\
\hline $\begin{array}{l}\text { Race, no. (\%) } \\
\text { Chinese } \\
\text { Malay } \\
\text { Indian } \\
\text { Others }\end{array}$ & $\begin{array}{l}59(79.7) \\
6(8.1) \\
8(10.8) \\
1(1.4)\end{array}$ & $\begin{array}{l}45(81.8) \\
5(9.1) \\
5(9.1) \\
0(0.0)\end{array}$ & $\begin{array}{c}14(73.7) \\
1(5.3) \\
3(15.8) \\
1(5.3)\end{array}$ & 0.292 \\
\hline $\begin{array}{l}\text { Non-smoker, no. (\%) } \\
\text { Ex-smoker, no. (\%) } \\
\text { Smoker, no. (\%) }\end{array}$ & $\begin{array}{l}42(56.8) \\
21(28.4) \\
11(14.9)\end{array}$ & $\begin{array}{l}34(61.8) \\
13(23.6) \\
8(14.6)\end{array}$ & $\begin{array}{l}8(42.1) \\
8(42.1) \\
3(15.8)\end{array}$ & 0.226 \\
\hline \multicolumn{5}{|l|}{ Symptoms at baseline } \\
\hline $\begin{array}{l}\text { Cough, no. }(\%) \\
\text { Not at all/ rarely } \\
\text { Occasionally but not bothersome } \\
\text { Most days } \\
\text { Severe and interferes with activity }\end{array}$ & $\begin{array}{c}24(32.4) \\
31(41.9) \\
19(25.7) \\
0(0)\end{array}$ & $\begin{array}{c}20(36.4) \\
21(38.2) \\
14(25.6) \\
0(0)\end{array}$ & $\begin{array}{c}4(21.1) \\
10(52.6) \\
5(26.3) \\
0(0)\end{array}$ & 0.489 \\
\hline Dyspnoea, no. (\%) & $64(86.5)$ & $45(81.8)$ & $19(100.0)$ & 0.056 \\
\hline $\begin{array}{l}\text { MMRC score, no. (\%) } \\
0 \\
1 \\
2 \\
3 \\
4\end{array}$ & $\begin{array}{l}10(14.3) \\
35(54.7) \\
14(21.9) \\
4(6.3) \\
1(1.4)\end{array}$ & $\begin{array}{c}8(15.7) \\
26(57.8) \\
8(17.8) \\
3(6.7) \\
0(0)\end{array}$ & $\begin{array}{l}2(10.5) \\
9(47.4) \\
6(31.6) \\
1(5.3) \\
1(5.3)\end{array}$ & 0.403 \\
\hline MMRC, median (IQR) & $1(0,3)$ & $1(0,3)$ & $1(1,2)$ & 0.314 \\
\hline \multicolumn{5}{|l|}{ Comorbidities } \\
\hline Diabetes mellitus, no. (\%) & $23(31.1)$ & $16(29.1)$ & $7(36.8)$ & 0.572 \\
\hline Hypertension, no. (\%) & $34(46.0)$ & $22(40.0)$ & $12(63.2)$ & 0.110 \\
\hline Hyperlipidaemia, no. (\%) & $43(58.1)$ & $31(56.4)$ & $12(63.2)$ & 0.788 \\
\hline Ischaemic heart disease, no. (\%) & $17(23.0)$ & $11(12.6)$ & $6(31.6)$ & 0.349 \\
\hline Asthma, no. (\%) & $2(2.5)$ & $2(3.6)$ & $0(0)$ & 0.399 \\
\hline \multicolumn{5}{|l|}{ Pulmonary physiology } \\
\hline Baseline FVC, mean (SD), L & $2.18(0.61)$ & $2.19(0.61)$ & $2.15(0.61)$ & 0.793 \\
\hline Baseline FVC \% predicted, mean (SD) & $71.5(15.0)$ & $71.4(14.8)$ & $71.5(16.1)$ & 0.993 \\
\hline Baseline FEV1, mean (SD), L & $1.89(0.50)$ & $1.90(0.51)$ & $1.88(0.50)$ & 0.867 \\
\hline Baseline FEV1 \% predicted, mean (SD) & $86.8(18.7)$ & $86.4(18.9)$ & $87.6(18.5)$ & 0.826 \\
\hline Baseline DLCO, mean (SD), $\mathrm{mM} / \mathrm{min} / \mathrm{kPa}^{\mathrm{a}}$ & $4.93(2.23)$ & $5.05(2.34)$ & $4.55(1.86)$ & 0.443 \\
\hline Baseline DLCO, \% predicted, mean (SD) ${ }^{\mathrm{a}}$ & $60.0(15.0)$ & $61.0(15.8)$ & $57.3(12.5)$ & 0.395 \\
\hline Baseline TLC, mean (SD), L & $3.80(0.83)$ & $3.81(0.86)$ & $3.76(0.75)$ & 0.848 \\
\hline Baseline TLC \% predicted, mean (SD) ${ }^{\mathrm{b}}$ & $79.1(15.9)$ & $80.2(16.7)$ & $75.3(12.5)$ & 0.310 \\
\hline
\end{tabular}

CTD-ILD: connective tissue disease related interstitial lung disease; DLCO: diffusing capacity for carbon monoxide; FEV1: forced expiratory volume in 1 second; FVC: forced vital capacity; HP: hypersensitivity pneumonitis; LAM: lymphangioleiomyomatosis; MMRC: minimal clinically important difference; PR: pulmonary rehabilitation; SD: standard deviation; TLC: total lung capacity

${ }^{a}$ Missing data: 10

${ }^{\mathrm{b}}$ Missing data: 14 
Table 1. Clinical characteristics, lung function and KBILD scores of patients followed up at an interstitial lung disease clinic in Singapore, grouped by participation in pulmonary rehabilitation (Cont'd)

\begin{tabular}{|c|c|c|c|c|}
\hline & $\begin{array}{c}\text { All } \\
\mathbf{n}=74\end{array}$ & $\begin{array}{l}\text { Declined to participate in PR } \\
\qquad n=55\end{array}$ & $\begin{array}{l}\text { Participated in PR } \\
\qquad \mathrm{n}=19\end{array}$ & $P$ value \\
\hline \multicolumn{5}{|l|}{ Diagnostic procedures } \\
\hline Bronchoalveolar lavage, no. (\%) & $28(37.8)$ & $22(40.0)$ & $6(31.6)$ & 0.591 \\
\hline Transbronchial lung biopsy, no. (\%) & $12(42.9)$ & $10(45.5)$ & $2(33.3)$ & 0.595 \\
\hline Surgical lung biopsy, no. (\%) & $4(5.4)$ & $3(5.5)$ & $1(5.3)$ & 1.00 \\
\hline \multicolumn{5}{|l|}{ Final diagnosis } \\
\hline $\begin{array}{l}\text { Idiopathic interstitial pneumonia, no. (\%) } \\
\text { CTD-ILD } \\
\text { HP } \\
\text { LAM } \\
\text { Unclassifiable }\end{array}$ & $\begin{aligned} 44 & (59.5) \\
24 & (32.4) \\
2 & (2.7) \\
2 & (2.7) \\
2 & (2.7)\end{aligned}$ & $\begin{aligned} 28 & (50.9) \\
21 & (38.2) \\
2 & (3.6) \\
2 & (3.6) \\
2 & (3.6)\end{aligned}$ & $\begin{array}{c}16(84.2) \\
3(15.8) \\
0(0) \\
0(0) \\
0(0)\end{array}$ & 0.209 \\
\hline \multicolumn{5}{|l|}{ Ongoing treatments } \\
\hline Corticosteroids, no. $(\%)$ & $30(40.5)$ & $23(41.8)$ & $7(36.8)$ & 0.460 \\
\hline Steroid sparing agents, no. (\%) & $24(32.4)$ & $18(32.7)$ & $6(31.6)$ & 0.582 \\
\hline Opioids, no. (\%) & $5(6.8)$ & $3(5.5)$ & $2(10.5)$ & 0.382 \\
\hline Long-term oxygen therapy, no. (\%) & $7(9.5)$ & $3(5.5)$ & $4(21.1)$ & 0.067 \\
\hline \multicolumn{5}{|l|}{ KBILD scores } \\
\hline Baseline total score, mean (SD) & $64.1(14.5)$ & $71.1(9.4)$ & $43.9(2.8)$ & $<0.001$ \\
\hline Follow-up total score, mean (SD) & $64.1(4.8)$ & $64.9(5.0)$ & $61.5(3.0)$ & 0.006 \\
\hline Change in total score, mean (SD) & $-0.1(14.1)$ & $-6.1(10.9)$ & $17.5(3.8)$ & $<0.001$ \\
\hline $\begin{array}{l}\text { Follow-up dyspnoea scores, mean (SD) } \\
\text { (Questions } 1,4,11,13 \text { ) }\end{array}$ & $13.6(2.8)$ & $12.9(2.9)$ & $13.6(2.2)$ & 0.305 \\
\hline $\begin{array}{l}\text { Change in dyspnoea scores, mean (SD) } \\
\text { (Questions } 1,4,11,13 \text { ) }\end{array}$ & $0.4(5.4)$ & $-2.0(3.8)$ & $7.3(2.7)$ & $<0.001$ \\
\hline $\begin{array}{l}\text { Baseline psychological scores, mean (SD) } \\
\text { (Questions 3,5,6,8,10,12,14) }\end{array}$ & $33.1(7.4)$ & $36.6(4.9)$ & $22.9(2.2)$ & $<0.001$ \\
\hline $\begin{array}{l}\text { Follow-up psychological scores, mean (SD) } \\
\text { (Questions 3,5,6,8,10,12,14) }\end{array}$ & $32.8(3.0)$ & $33.4(3.2)$ & $31.1(1.7)$ & 0.003 \\
\hline Change in psychological scores, mean (SD) & $-0.3(7.2)$ & $-3.2(5.9)$ & $8.1(2.6)$ & $<0.001$ \\
\hline $\begin{array}{l}\text { Baseline chest symptoms scores, mean (SD) } \\
\text { (Questions 2,7,9) }\end{array}$ & $15.8(3.6)$ & $17.1(3.1)$ & $11.9(1.4)$ & 0.003 \\
\hline $\begin{array}{l}\text { Follow-up chest symptoms scores, mean (SD) } \\
\text { (Questions } 2,7,9)\end{array}$ & $15.0(3.0)$ & $15.5(3.0)$ & $13.4(2.6)$ & 0.008 \\
\hline Change in chest symptoms scores, mean (SD) & $-0.8(3.9)$ & $-1.6(4.0)$ & $1.5(2.7)$ & 0.002 \\
\hline
\end{tabular}

CTD-ILD: connective tissue disease related interstitial lung disease; DLCO: diffusing capacity for carbon monoxide; FEV1: forced expiratory volume in 1 second; FVC: forced vital capacity; HP: hypersensitivity pneumonitis; LAM: lymphangioleiomyomatosis; MMRC: minimal clinically important difference; PR: pulmonary rehabilitation; SD: standard deviation; TLC: total lung capacity

${ }^{a}$ Missing data: 10

${ }^{\mathrm{b}}$ Missing data: 14 
sparing agents, opioids and long-term oxygen did not differ significantly between study arms, suggesting that observed differences in KBILD scores and 6MWT distances were not due to differences in therapies received. Our study highlighted that there are measurable and important benefits for ILD patients who undergo PR. The study showed that while the KBILD was validated in a culturally distinct population, it remained robust and sensitive to changes in the health status of ILD patients.

There are limitations to our research. Firstly, as a single-centre study, the small sample sizes limit the external applicability of our findings. Secondly, our study did not address barriers and facilitators for PR uptake. A single-centre study within a district general hospital showed that a lack of awareness and low perceived benefits were important barriers to $\mathrm{PR},{ }^{10}$ making PR uptake an area for our future research. Thirdly, the KBILD was conceptualised as a selfadministered instrument, but some of our patients completed it with assistance from a translator. As the KBILD has yet to be validated for local languages, the current study would not have been possible without translators. A literature review conducted did not identify prior local studies describing the use of KBILD or Saint George's Respiratory Questionnaire in ILD patients. We had chosen the KBILD as it was shorter and easier to administer. Finally, enrolment into PR was low, which may have introduced selection bias, although symptom management was important in improving the QoL of ILD patients. Our study suggests that PR produces measurable improvements based on a patient-reported instrument, and encourages clinicians to continue to refer ILD patients for PR.

Patients who participated in PR demonstrated improvements in KBILD scores and 6MWT distances, consistent with published observations that PR improves QoL and exercise capacity. The KBILD is a robust instrument that has been validated in various European settings. Efforts should be taken to translate and validate it for use in Singapore.

\section{REFERENCES}

1. Travis WD, Costabel U, Hansell DM, et al. An official American Thoracic Society/European Respiratory Society statement: Update of the international multidisciplinary classification of the idiopathic interstitial pneumonias. Am J Respir Crit Care Med 2013;188:733-48.

2. Carvajalino S, Reigada C, Johnson MJ, et al. Symptom prevalence of patients with fibrotic interstitial lung disease: a systematic literature review. BMC Pulm Med 2018;18:78.

3. Belkin A, Swigris JJ. Health-related quality of life in idiopathic pulmonary fibrosis: where are we now? Curr Opin Pulm Med 2013;19:474-79

4. Nakazawa A, Cox NS, Holland AE. Current best practice in rehabilitation in interstitial lung disease. Ther Adv Respir Dis 2017;11:115-28.

5. Dowman L, Hill CJ, Holland AE. Pulmonary rehabilitation for interstitial lung disease. Cochrane Database Syst Rev 2014; CD006322.

6. Patel AS, Siegert RJ, Brignall K, et al. The development and validation of the King's Brief Interstitial Lung Disease (K-BILD) health status questionnaire. Thorax 2012;67:804-10.

7. Wapenaar M, Patel AS, Birring SS, et al. Translation and validation of the King's Brief Interstitial Lung Disease (K-BILD) questionnaire in French, Italian, Swedish, and Dutch. Chron Respir Dis 2017; 14:140-50.

8. Sinha A, Patel AS, Siegert RJ, et al. The King's Brief Interstitial Lung Disease (KBILD) questionnaire: an updated minimal clinically important difference. BMJ Open Respir Res 2019;6:e000363.

9. du Bois RM, Weycker D, Albera C, et al. Six-minute-walk test in idiopathic pulmonary fibrosis: test validation and minimal clinically important difference. Am J Respir Crit Care Med 2011;183:1231-37.

10. McCarron EP, Bailey M, Leonard B, et al. Improving the uptake: Barriers and facilitators to pulmonary rehabilitation. Clin Respir J 2019;13:624-29.

Yi Hern Tan, ${ }^{1}$ MRCP (Edin), Mariya Mohd Nor, ${ }^{1}$

Michelle Li Wei Kam, ${ }^{1} M R C P\left(\right.$ Edin), Su Ying Low, ${ }^{1}{ }_{M R C P}(U K)$

\footnotetext{
${ }^{1}$ Department of Respiratory and Critical Care Medicine, Singapore General Hospital, Singapore
}

Correspondence: Dr Yi Hern Tan, Department of Respiratory and Critical Care Medicine, Singapore General Hospital, Academia Building, 20 College Road, Singapore 169856.

Email: tan.yi.hern@singhealth.com.sg 